\title{
ANÁLISE DE ATRIBUTOS NA PREFERÊNCIA ENTRE CONSUMO DE MEDICAMENTOS GENÉRICOS E SIMILARES OU MEDICAMENTOS DE REFERÊNCIA
}

\section{ANALYSIS OF ATTRIBUTES IN THE PREFERENCE BETWEEN CONSUMPTION OF GENERIC DRUGS AND SIMILAR OR REFERENCE DRUGS}

\author{
MARCOS ROBERTO LUPPE \\ Escola de Artes, Ciências e Humanidades - Universidade de São Paulo (EACH/USP) \\ Doutor em Administração (FEA-USP) \\ Orcid: http://orcid.org/0000-0003-2297-1900 / E-mail: mluppe@usp.br
}

Rua Arlindo Bettio, 1000 Prédio I1 Sala 303C - Ermelino Matarazzo, São Paulo - SP, 03828-000

\section{CAROLINE MEDEIROS ROSSI}

Escola de Artes, Ciências e Humanidades - Universidade de São Paulo (EACH/USP)

Discente do curso de Marketing na EACH/USP

Orcid: http://orcid.org/0000-0002-6921-5347 / E-mail: caroline.mrossi@gmail.com

\section{ROSANE RIVERA TORRES}

Escola de Artes, Ciências e Humanidades - Universidade de São Paulo (EACH/USP)

Doutor em Administração (FEA-USP)

Orcid: http://orcid.org/0000-0002-0286-3972 / E-mail: rosanetorres@usp.br

HELDER DE SOUZA AGUIAR

Faculdade de Tecnologia de Bragança Paulista (FATEC)

Doutor em Administração (FEA-USP)

Orcid: http://orcid.org/0000-0003-2919-4315 / E-mail: helder.aguiar@fatec.sp.gov.br

\section{RESUMO}

O objetivo principal do estudo foi analisar a importância dos atributos marca, qualidade e preço no processo de decisão de compra dos consumidores de medicamentos genéricos, similares e de referência na cidade de São Paulo e nos municípios da região metropolitana. Definiu-se para a coleta de dados o método de pesquisa quantitativo do tipo survey, via plataforma online, com amostragem não probabilística por conveniência. Os resultados indicam que $76,4 \%$ dos respondentes da pesquisa têm conhecimento da diferença entre esses medicamentos. Quanto as motivações que levam a preferência por esses tipos de medicamentos, preço, qualidade e confiança são os principais motivadores para a compra de medicamentos genéricos e similares, e qualidade, confiança e recomendação médica para os medicamentos de referência. Analisadas as motivações quando os respondentes realizam a compra para outra pessoa (shopper), aproximadamente $60 \%$ afirmaram ter preferência por medicamentos genéricos ou similares, $31 \%$ por medicamentos de referência e os demais declararam ser indiferentes ao tipo de medicamento. Em paralelo, a influência de profissionais da saúde na decisão de compra dos consumidores, quanto à recomendação médica ou à recomendação do balconista da farmácia, também se mostrou expressiva, resultados esses semelhantes aos obtidos em outros estudos relacionados ao tema deste trabalho.

Palavras-chave: Comportamento do consumidor. Tipos de medicamentos. Processo de decisão de compra. 


\begin{abstract}
The main objective of the study was to analyze the importance of brand, quality and price attributes in the buying decision process of generic, similar and reference drugs in the city of São Paulo and in the metropolitan area. The quantitative survey method was defined for the data collection, via online platform, with non-probabilistic sampling for convenience. The results indicate that $76,4 \%$ of the respondents are aware of the difference between these drugs. As for the motivations that lead to preference for these types of drugs, price, quality and trust are the main motivators for the purchase of generic and similar drugs, and quality, trust and medical recommendation for reference drugs. When the motivations were analyzed when the respondents made the purchase to another person (shopper), approximately $60 \%$ said they had a preference for generic or similar drugs, 31\% for reference drugs and the others stated they were indifferent to the type of drug. At the same time, the influence of health professionals on consumers' purchase decisions, regarding the medical recommendation or the recommendation of the pharmacy clerk, was also expressive, results similar to those obtained in other studies related to the theme of this study.
\end{abstract}

Keywords: Consumer behavior. Types of drugs. Buying decision process.

\title{
1 INTRODUÇÃO
}

Nos últimos anos, a indústria farmacêutica apresentou grande crescimento e evolução no Brasil, aproveitando-se da expansão dos gastos do mercado de saúde e do aumento da renda doméstica das famílias (PWC, 2014). Segundo estimativas da consultoria IMS Health, o mercado farmacêutico poderá atingir o valor de 1,4 trilhões de dólares até 2020 e o Brasil passará a ocupar a quinta posição no ranking mundial deste mercado (INTERFARMA, 2016).

A Federação Brasileira das Redes Associativistas e Independentes de Farmácias (Febrafar) com base na auditoria Intercontinental Medical Statistics (IMS Health, 2015) divulgou a previsão para o mercado farmacêutico brasileiro de varejo de que este dobrará de tamanho em cinco anos, com a estimativa para 2017 de 87,0 bilhões de reais em vendas (INTERFARMA, 2016).

Até julho de 2017, com base no acumulado dos últimos 12 meses, o mercado farmacêutico no Brasil apresentou crescimento de 6,7\% em comparação a 2016, o que correspondente a 54,3 bilhões de reais (SINDUSFARMA, 2017). De acordo a classificação de medicamentos pela Agência Nacional de Vigilância Sanitária - ANVISA, para o ano de 2016, os medicamentos similares corresponderam a $45,5 \%$ do mercado total, os medicamentos referências a $28,1 \%$ e os medicamentos genéricos corresponderam a $26,4 \%$ (INTERFARMA, 2016).

As políticas públicas para acesso aos medicamentos no Brasil, com destaque para a Lei dos Medicamentos Genéricos, Lei no 9.787 de 10 de fevereiro de 1999, ampliaram a concessão de registros de medicamentos considerados de menor custo, incentivando assim as empresas a registrarem as versões genéricas de fármacos com patentes expiradas (ANVISA,1999). Paralelamente a Lei dos Medicamentos Genéricos, a ANVISA estabeleceu ações para a regulamentação das versões genéricas e similares dos medicamentos referência, de acordo com requisitos relacionados aos conceitos de bioequivalência e intercambialidade (ANVISA, 2003).

Nota-se que o mercado farmacêutico vem apresentando amplo crescimento nos últimos anos, com destaque para o desempenho dos medicamentos genéricos e similares. Desta 
forma, analisar a importância de atributos que influenciam a decisão de compra destes tipos de medicamentos, como, por exemplo, os atributos marca, qualidade e preço, escolhidos para realização deste estudo, se faz relevante diante do cenário de crescimento e das perspectivas para o mercado farmacêutico, em especial, dos medicamentos genéricos e similares, no que tange ao processo de compra de medicamentos. Esses três atributos foram escolhidos para serem avaliados em virtude da importância dos mesmos em outros estudos relacionados a temática deste trabalho (FERNANDES; COUTINHO; VALLE, 2011; SANYAL; DATTA; BANERJEE, 2011; BLATT et al. 2012; SOUSA; MESQUITA; LARA, 2013; DUNNE; DUNNE, 2015; GUTTIER et al., 2016; FERREIRA et al., 2017).

Logo, por meio deste estudo, pretende-se como objetivo principal analisar a importância dos atributos marca, qualidade e preço no processo de decisão de compra dos consumidores de medicamentos genéricos, similares e de referência na cidade de São Paulo e nos municípios da região metropolitana.

Desdobram-se os seguintes objetivos secundários: verificar o conhecimento dos consumidores residentes na grande São Paulo a respeito das diferenças entre esses tipos de medicamentos, analisar o comportamento do shopper na compra desses medicamentos, verificar a influência de profissionais da saúde na decisão de compra desses medicamentos e por fim analisar se o perfil socioeconômico dos entrevistados interfere nas escolhas desses medicamentos.

A próxima seção será destinada à fundamentação teórica que alicerça o desenvolvimento do trabalho, contendo a revisão da literatura. Em seguida, serão abordados os tópicos relacionados à pesquisa, como o método, o universo de pesquisa, a definição da amostra e a coleta de dados. Na sequência, são apresentados os resultados obtidos nas pesquisas realizadas e, por fim, apresentadas as conclusões, limitações e possíveis extensões do presente estudo.

\section{FUNDAMENTAÇÃO TEÓRICA}

\subsection{DECISÃO DE COMPRA DE MEDICAMENTOS}

O comportamento do consumidor consiste em um conjunto de referências que servem de base para o conhecimento das culturas, valores, crenças e desejos. Deste modo, para compreender o processo de compra é necessário a identificação dos influenciadores desse processo. O objetivo fundamental do marketing é atender e satisfazer as necessidades e desejos dos consumidores e, para que isso seja possível, é imprescindível entender e compreender o comportamento do consumidor (KOTLER; KELLER, 2012).

A administração de marketing no que tange o processo de decisão de compra envolve o reconhecimento de necessidades, busca de informações, avaliação de alternativas, decisão de compra e o comportamento pós-compra. Especificamente no estágio de decisão de compra, os consumidores utilizam critérios para escolher produtos ou serviços e, consequentemente, as marcas, os quais podem variar de acordo com a situação. Neste cenário, a decisão de compra sofre influência de fatores situacionais, isto é, do risco percebido pelo cliente em relação ao objeto de compra (BLACKWELL; MINIARD; ENGEL, 2005; SOLOMON, 2016).

A decisão de compra não é mais considerada uma atitude passiva do consumidor em aceitar ou não uma proposta que lhe é oferecida. Agora, de forma geral, os consumidores participam ativamente da construção das ofertas que the são oferecidas pelas empresas 
(COBRA; BREZZO, 2010). A percepção de estímulos pelos consumidores é influenciada pelas características intrínsecas aos mesmos e a decisão de compra influenciada por estes estímulos, determina o tipo de comportamento de compra que será realizado (SOLOMON, 2016).

A adequada qualificação dos produtos e serviços pelas empresas depende significativamente do conhecimento das variáveis de influência sobre o comportamento de compra dos consumidores (BLACKWELL; MINIARD; ENGEL, 2005, SOLOMON, 2016). Assim, as estratégias de marketing devem ser orientadas para o mercado-alvo pretendido, alinhado ao modo de tomada de decisão destes consumidores-alvo. Conforme será abordado a seguir e nos próximos tópicos da revisão da literatura, diferentes autores indicam que a tomada de decisão de medicamentos é influenciada por diferentes atributos, os quais atuam diretamente no processo de decisão de compra dos consumidores, tendo influências positivas e negativas, de acordo a percepção dos benefícios e valores destes em relação aos medicamentos que pretendem adquirir.

No mercado farmacêutico, com relação à atitude e decisão de compra de consumidores de medicamentos, alguns fatores como idade, gênero, renda e o plano de saúde afetam a atitude de compra e, consequentemente, a escolha entre os medicamentos referência, genérico e similar (SANSGIRY; BHOSLE; POPE, 2005; GASPAR, 2016; SAMAOUI; KILANI; TOUZANI, 2016; ZERBINI; VERGURA; LUCERI, 2017). De modo geral, consumidores mais jovens e com maior nível de instrução apresentam maior tendência em realizar essa substituição (GAITHER et al., 2001).

Pesquisas com consumidores de medicamentos genéricos revelaram que experiências negativas anteriores com estes produtos que manifestaram maior grau de risco percebido influenciaram negativamente a atitude em relação a este tipo de medicamentos. Estes estudos também revelaram que a complexidade, a condição e a gravidade da doença também foram fatores de influência na decisão de compra dos consumidores em usar genéricos. Ainda, os consumidores possuem maior preferência por medicamentos referência para o tratamento de doenças crônicas, como asma, diabetes e problemas cardíacos (GAITHER et al., 2001; SANSGIRY; BHOSLE; POPE, 2005).

A atitude e a decisão de compra dos consumidores também são influenciadas pelas estratégias de marketing das empresas farmacêuticas (SANSGIRY; BHOSLE; POPE, 2005; GASPAR, 2016; SAMAOUI; KILANI; TOUZANI, 2016), no que diz respeito às estratégias de preços e de marca e também em relação a promoção de diferenciais dos produtos, por exemplo, a qualidade (GILL et al., 2010; GASPAR, 2016; FERREIRA et al., 2017; ZERBINI; VERGURA; LUCERI, 2017). Nota-se que a divulgação e promoção de medicamentos genéricos e de similares é falha e concentrada especialmente para os medicamentos referência (SANSGIRY; BHOSLE; POPE, 2005; GASPAR, 2016; SAMAOUI; KILANI; TOUZANI, 2016; ZERBINI; VERGURA; LUCERI, 2017). Esse foco da promoção de medicamentos referência mostrou ser eficaz para o reconhecimento das marcas pelos consumidores (MINTZES et al., 2003; SANYAL; DATTA, 2011; FERREIRA et al., 2017).

Em paralelo, os profissionais da saúde também são fortes influenciadores da atitude e da decisão de compra dos consumidores de medicamentos (SANYAL; DATTA, 2011; DUNNE; DUNNE, 2015; FERREIRA et al., 2017; SANYAL; DATTA; BANERJEE, 2017). São, portanto, o gatilho para o direcionamento da compra de genéricos e similares em substituição aos medicamentos referência. A mídia também tem influenciado positivamente a compra de medicamentos de marca pelos consumidores (SANSGIRY; BHOSLE; POPE, 2005; FERREIRA et al., 2017). 
Consumidores que possuem uma postura participativa com seus cuidados de saúde e que são ativos na discussão, junto a profissionais da saúde a respeito de questões de serviços clínicos e de custos de medicamentos, apresentam maior tendência em substituir medicamentos referência por opções de menor custo, genéricas e similares (MURRAY et al., 2003). Em linha, operadoras de saúde, médicos e farmacêuticos são os principais agentes estimuladores da substituição de medicamentos referência pelos genéricos e similares, podendo auxiliar na melhora da percepção destes produtos, influenciando, assim, diretamente, a atitude e o processo de decisão de compra destes consumidores (SANSGIRY; BHOSLE; POPE, 2005; FERREIRA et al., 2017).

Como pretende-se analisar a importância dos atributos marca, qualidade e preço no processo de decisão de compra dos consumidores de medicamentos genéricos, similares e de referência, a base conceitual relacionada a esses atributos será abordada nos próximos tópicos.

\subsection{ATRIBUTO MARCA}

Uma marca é um bem ou serviço que agrega dimensões que o diferenciam de outros produtos desenvolvidos para satisfazer a mesma necessidade. Ainda, estas diferenças podem ser intrínsecas e tangíveis, relacionadas ao desempenho do produto, ou extrínsecas e intangíveis, relacionadas a representações ou significados em termos abstratos (KOTLER; KELLER, 2012). As marcas desempenham diferentes papéis de acordo a perspectiva dos consumidores ou fabricantes. Elementos de marcas são recursos que podem ser registrados e servem para identificar e diferenciar uma marca (KELLER; MACHADO, 2006; SERRALVO, 2008).

As marcas fortes podem definir o produto escolhido pelo consumidor e diferenciá-lo frente aos outros produtos numa avaliação, quando este consumidor se encontra em um cenário com informações e experiências anteriores limitadas, reduzindo assim os riscos e incertezas da compra (HOEFFLER; KELLER, 2003). As empresas devem usar as marcas para informar aos consumidores sobre o seu posicionamento e sobre alguns atributos do produto atrelados a marca, como a qualidade e credibilidade (ERDEM; SWAIT, 1998). Segundo Cunha (1997), a marca interfere na intenção de compra dos consumidores. As marcas genéricas, como a de medicamentos genéricos, são consideradas fracas em termos de identificação, memorização e poder de venda, já que não apresentam aspectos de diferenciação, que são característicos de uma marca (HOEFFLER; KELLER, 2003).

No caso do mercado farmacêutico, os medicamentos referência têm por fortaleza as suas marcas, que remetem diretamente a atributos tais como qualidade e confiança. A marca possui grande influência no que tange ao business-to-business, no caso a relação entre os laboratórios farmacêuticos e os profissionais da saúde, para os quais a propaganda é direta e permitida para os medicamentos de prescrição (SPITERI; DION, 2004). Desta forma, os profissionais da saúde também podem influenciar o processo de compra de medicamentos dos consumidores finais, em especial no caso de medicamentos de prescrição (SANYAL; DATTA, 2011; DUNNE; DUNNE, 2015; FERREIRA et al., 2017; SANYAL; DATTA; BANERJEE, 2017).

Os medicamentos originadores (referência) apresentam preço superior aos genéricos e similares em virtude do custo do desenvolvimento da tecnologia que gera a patente e, consequentemente, a marca. $O$ preço de medicamentos referência, mesmo após a expiração da patente, permanece semelhante à fase pré-patente ou até superior. Segundo Skipper e 
Vejlin (2015), os consumidores que são leais à marca, são insensíveis ao preço mesmo após a disponibilização de outras versões de menor preço.

Segundo Sanyal e Datta (2011), Dunne e Dunne (2015) e Ferreira et al. (2017), no caso de medicamentos genéricos e similares, a ideia de uma marca também pode estar atrelada ao nome do laboratório detentor do registro do produto e ao local de fabricação. Os similares também apresentam marcas, porém, de modo geral, estas não possuem o mesmo apelo e força que as marcas dos medicamentos referência, principalmente pelo fato da baixa credibilidade dos consumidores em relação a qualidade destes produtos. Esses mesmos autores indicam que a ausência de uma marca para a versão genérica de medicamentos, aliada ao desconhecimento de grande parte dos consumidores sobre a regulamentação destes produtos, ocasiona a percepção de qualidade inferior em comparação ao fármaco de referência. Além disso, o pouco estímulo a este tipo de medicamentos pela classe médica, aumenta ainda mais o ceticismo e a insegurança por parte dos consumidores. Contudo, o cenário econômico desafiador, a maior divulgação sobre aspectos de qualidade de medicamentos e o incentivo a adesão a tratamentos de longa duração tem auxiliado na ampliação do consumo de medicamentos genéricos e similares, em virtude do baixo custo em relação aos fármacos originadores (GILL et al., 2010).

\subsection{ATRIBUTO QUALIDADE}

Nos últimos anos, os consumidores vêm mudando o seu modo de pensar, analisar e perceber os produtos e serviços, no que diz respeito ao processo de decisão de compra, incluindo aqui a intenção de compra (ALMEIDA; BOTELHO, 2005). A American Society for Quality Control define qualidade como a totalidade dos atributos e características de um produto que afetam a sua capacidade de satisfazer necessidades declaradas ou implícitas. No relacionamento entre o cliente e a empresa, a confiança está diretamente relacionada com o atributo qualidade e tem papel fundamental na promoção da cooperação entre as partes, isto é, à fidelização do cliente a marca (SANTOS; ROSSI, 2002).

A qualidade percebida pode ser definida como o julgamento do consumidor a respeito da excelência ou da superioridade geral do produto, a qual é composta de características as quais os consumidores atribuem importância relativa e que satisfazem necessidades ou expectativas dos mesmos (ZEITHAML, 1988; LIMA; STEINER NETO, 2013). Estas características de produtos ou serviços podem ser percebidas pelos consumidores como benefícios e, desta forma, agregam valor às marcas (ALMEIDA; BOTELHO, 2005). Sob outra perspectiva, a qualidade percebida fundamentada em uma diferença de preço consiste, numa percepção empregada pelos consumidores, em situações que envolvem o processo de tomada de decisão, em uma compra na qual as informações sobre o produto ou serviço que se pretende adquirir é limitada (URBANY et al., 1997).

Existe uma relação entre a qualidade, seja de produtos ou serviços, satisfação de clientes e lucratividade das empresas, de modo que níveis elevados de qualidade resultam em níveis mais elevados de satisfação de clientes, que também justificam preços mais altos para os produtos e serviços identificados como de alta qualidade. A qualidade é a chave para a criação de valor e para a satisfação do cliente e alguns princípios devem ser seguidos para a entrega da alta qualidade aos clientes-alvo nas etapas anterior, durante e posterior a compra (KOTLER; KELLER, 2012; YANAZE, 2011). Segundo Zeithaml (1988), a qualidade e a intenção de compra estão diretamente relacionadas e o atributo qualidade será o determinante da fidelização ou não fidelização do cliente a uma marca. 
No Brasil, a qualidade de medicamentos genéricos e similares é regulamentada por meio do conceito de bioequivalência em relação ao fármaco originador do medicamento de referência (GUTTIER et al., 2016; FERREIRA et al., 2017). Esta regulamentação torna pouco efetiva a discussão de méritos e controle de qualidade (FONSECA, 2014). Ainda, alguns fatores como a credibilidade atribuída aos medicamentos referência, a bioequivalência não comprovada de alguns medicamentos genéricos e similares (DUNNE; DUNNE, 2015; FITZGERALD; JACOBSON, 2011) e também a consideração negativa da mídia à falta de controle de qualidade de algumas empresas farmacêuticas nacionais (ALISKI, 2014; BARATA-SILVA et al., 2017), reduzem ainda mais a credibilidade de profissionais da saúde e consumidores com relação aos medicamentos genéricos e similares.

A qualidade para medicamentos é essencialmente um fator de uso, porém, pelo fato destes produtos estarem relacionados à saúde das pessoas, o risco envolvido é alto (MOSS, 2007). Logo, a menor qualidade de medicamentos é percebida por consumidores e profissionais da saúde como envolvendo maior risco, em comparação a vantagem de preço. Assim, tanto os consumidores como os profissionais da saúde ao acreditarem que a eficácia de medicamentos genéricos e similares é menor em comparação ao de referência, estes são menos propensos a efetuar a troca pelos genéricos e similares. Profissionais da saúde possuem conhecimento a respeito da experiência do uso de medicamentos genéricos e similares, ao passo que os consumidores usam de pistas extrínsecas como o comparativo de preço, o nome do laboratório, o país de origem do produto e a marca, caso seja aplicável, para analisar a qualidade percebida destes tipos de medicamentos, buscando reduzir a sensação de risco atrelada ao uso destes produtos (SANYAL; DATTA, 2011; DUNNE; DUNNE, 2015; GUTTIER et al., 2016; FERREIRA et al., 2017; SANYAL; DATTA; BANERJEE, 2017).

Esses mesmos autores indicam que no relacionamento entre profissionais da saúde e consumidores, a classe médica tem estimulado pouco o uso de medicamentos genéricos e similares por meio da prescrição, o que atrapalha a percepção de qualidade pelos consumidores por meio da experiência no uso destes tipos de medicamentos. Em contrapartida, os farmacêuticos têm enfrentado a resistência da classe médica a prescrição de genéricos e similares e também o desconhecimento dos consumidores sobre a regulamentação de qualidade destes medicamentos, o que contribui ainda mais para a permanência de barreiras quanto à discussão da qualidade percebida atrelada ao fator de uso e, consequentemente, a utilização de genéricos e similares em substituição aos medicamentos de referência (GILL et al.; 2010; GUTTIER et al., 2016).

\subsection{ATRIBUTO PREÇO}

O preço pode exercer a função de influenciar tanto a intenção e decisão de compra dos clientes, como as estratégias de marketing adotadas pelas organizações. Ainda, indica ao cliente o valor e a qualidade do produto ou serviço em relação às marcas dos competidores (HAN; GUPTA; LEHMANN, 2011).

A intenção e consequentemente decisão de compra dos clientes está fundamentada na percepção de preço pelos consumidores em conformidade a aquilo que consideram o verdadeiro preço corrente e não necessariamente o preço que é definido pelas empresas. Ainda, após as diversas crises econômicas, os consumidores passam a comprar mais por necessidade do que por desejo e, deste modo, passaram a optar mais por produtos de preço mais baixo. Desta forma, duas abordagens são consideradas pertinentes a este tema de pesquisa: preços de referência e inferências preço-qualidade (NAGLE; HOGAN, 2007). 
Conforme a abordagem de preços de referência, os consumidores não conseguem se lembrar precisamente de faixas de preços de produtos ou serviços. Logo, quando estão envolvidos numa compra, os consumidores adotam preços de referência, comparando assim um preço observado com um preço de referência interno de que se recordem ou em linha com a sua estrutura de referência externa. Já do ponto de vista da abordagem inferências preço-qualidade, de forma geral, os consumidores consideram o preço como indicador de qualidade de produtos ou serviços. Este indicativo também está relacionado a fatores posteriores a realização de uma compra, por exemplo, a confiança, a credibilidade e a fidelidade à marca (KOTLER; KELLER, 2012; SOLOMON, 2016).

Para os medicamentos genéricos e similares, preço e qualidade percebida são os atributos essenciais em relação à decisão de compra dos consumidores (FERNANDES; COUTINHO; VALLE, 2011; BLATT et al., 2012; SOUSA; MESQUITA; LARA, 2013; GUTTIER et al., 2016). Os consumidores brasileiros apresentam elevado nível de conhecimento dos preços mais baixos dos medicamentos genéricos e similares, quando comparados aos medicamentos de referência. Ainda, a opção por estes tipos de medicamentos é influenciada principalmente pelo baixo preço destes produtos (BLATT et al., 2012; SOUSA; MESQUITA; LARA, 2013; GUTTIER et al., 2016; FERREIRA et al., 2017).

Os medicamentos sejam genéricos, similares ou de referência, no que tange o processo de compra, apresentam elevado envolvimento do consumidor, e deste modo, buscando simplificar este processo, os consumidores tendem a tomar decisões que são mais racionais, remetendo à percepção do preço pelo consumidor (KAYTAZ; GUL, 2014). Em paralelo, a preocupação dos profissionais da saúde à adesão dos pacientes ao tratamento, também se constitui num importante fator de incentivo a busca de terapias de menor custo, viabilizadas pelo uso de medicamentos genéricos e similares, que são reconhecidos por apresentarem os menores preços (GILL et al., 2010; BLATT et al., 2012; SOUSA; MESQUITA; LARA, 2013; GUTTIER et al., 2016; FERREIRA et al., 2017).

Como já dito, o preço consiste num importante fator de consideração pelos consumidores para a compra de medicamentos. Em contrapartida, estudos recentes têm evidenciado que preços mais baixos não refletem uma qualidade inferior de medicamentos genéricos e similares, mas sim representam uma economia de custo e um incentivo ao uso destes tipos de medicamentos (KASWENGI; DIALLO, 2015; GUTTIER et al., 2016). Ainda, o incentivo a utilização deste tipo de medicamentos pelos profissionais da saúde tende a reduzir a percepção de que o baixo preço representa uma qualidade inferior frente aos medicamentos de referência (KOBAYASHI et al., 2011; SANYAL; DATTA, 2011; DUNNE; DUNNE, 2015; SANYAL; DATTA; BANERJEE, 2017). De modo geral os preços de medicamentos de referência permanecem em nível pré-patente ou tendem a subir, enquanto os medicamentos genéricos apresentam preço 35\% menor que o de referência e os similares apresentam preços intermediários (FERREIRA et al., 2017).

\section{MÉTODO DE PESQUISA}

Partindo-se da definição dos atributos marca, qualidade e preço, que influenciam o processo de decisão de compra de consumidores de medicamentos de referência, genéricos e similares, foi realizado um estudo empírico-analítico, conduzido por meio de pesquisa quantitativa do tipo conclusiva descritiva. Para Kinnear e Taylor (1999), a pesquisa conclusiva fornece informações que auxiliam o gestor a avaliar e selecionar uma ação. $O$ desenho da pesquisa é caracterizado por procedimentos formais, envolvendo objetivos de pesquisa 
claramente definidos. Segundo Zikmund e Babin (2012, p.52), "a pesquisa descritiva, como diz o nome, descreve características de objetos, pessoas, grupos, organizações ou ambientes".

A técnica de coleta de dados desenvolvida foi do tipo levantamento ou e-survey com caracterização temporal transversal única. Segundo Malhotra (2012, p.109), no corte transversal único "é extraído da população alvo uma amostra de entrevistados e as informações são obtidas uma única vez".

A população alvo desta pesquisa foi composta por consumidores de medicamentos da cidade de São Paulo e dos municípios da região metropolitana, com 18 anos ou mais e que haviam comprado medicamentos nos últimos 90 dias. Entretanto, para tornar viável a coleta de dados, optou-se por realizar uma amostra não probabilística por conveniência. Segundo Hair Jr et al. (2009), na amostra não probabilística, a seleção dos elementos que irão compor a amostra não é feita com o objetivo de ser estatisticamente representativa da população em questão. Ao contrário, o pesquisador utiliza métodos subjetivos para selecionar os elementos da amostra, fazendo com que a probabilidade de um elemento da população ser escolhido não seja conhecida. Ainda segundo os mesmos autores, a amostra por conveniência envolve a seleção de elementos que estejam mais disponíveis para participar do estudo e que tenham condições de fornecer as informações necessárias para a pesquisa. Cabe ainda ressaltar que esse tipo de amostragem não permite a generalização dos resultados, pois tem por objetivo somente descrever os achados para o grupo pesquisado.

\subsection{COLETA DE DADOS}

Para elaboração do questionário foram considerados os objetivos do estudo, o público-alvo e o método de pesquisa previamente determinado. Foi utilizado como base para o desenvolvimento do instrumento de coleta, o questionário de uma pesquisa de imagem, já validado, desenvolvido por uma empresa de estudos de mercado para um laboratório farmacêutico, cujo objetivo era avaliar a performance da marca do laboratório em questão e de seus concorrentes, diante dos públicos delimitados (farmácias independentes, balconistas, farmacêutico e consumidores). Obteve-se então, o questionário desta pesquisa composto por cinco blocos, a saber, totalizando 38 questões:

- Bloco 1: Composto por três questões filtro, em escala nominal, com o objetivo de selecionar a amostra para a aplicação da pesquisa.

- Bloco 2: Composto por três questões, em escala nominal, sobre o local de compra de medicamentos (localização, tipo e motivações para escolha de determinado estabelecimento).

- Bloco 3: Composto por vinte e uma questões, em escala nominal e ordinal, sobre o conhecimento dos entrevistados em relação aos tipos de medicamentos (genéricos, similares e de referência - de marca) e também sobre os fatores que influenciam a recomendação e a compra de determinadas marcas e tipos de medicamentos.

- Bloco 4: Composto por oito questões, em escala nominal e ordinal, sobre os fatores que influenciam o comportamento de compra de medicamentos de prescrição. Para medicamentos de prescrição considerou-se a influência direta do balconista/atendente de farmácia no processo de compra.

- Bloco 5: Composto por três questões, em escala nominal e ordinal, com a finalidade de caracterizar a amostra de acordo com as variáveis demográficas (sexo) e as variáveis socioeconômicas (nível educacional e renda). 
O questionário foi disponibilizado em uma plataforma online (Survey Monkey) através de um link para acesso em redes sociais (Facebook e WhatsApp). Inicialmente realizou-se um pré-teste com 15 respondentes por meio de questionários impressos durante o período de 02 a 12 de outubro de 2017, totalizando 10 dias de coleta. Com base no pré-teste, foram feitos ajustes na questão filtro referente à localização do respondente, nas questões sobre as motivações de escolha do tipo de farmácia, e sobre o laboratório dos medicamentos de referência, genéricos e similares mais utilizado. Após esta etapa, o questionário passou pela avaliação de dois especialistas com aproximadamente 10 anos de atuação na área estudada, tendo como objetivo a validação do conteúdo, sendo que os mesmos sugeriram pequenos ajustes no instrumento de coleta dos dados.

Dessa forma, depois de realizados os ajustes sugeridos pelos especialistas, o questionário definitivo foi disponibilizado aos respondentes durante o período de 29 de outubro e 03 de dezembro de 2017, totalizando 35 dias de coleta. Foram coletados 348 questionários, sendo válidos 220 questionários - respondidos completamente, o que representa uma taxa de aproveitamento de 63,2\%. Segundo Malhotra (2012, p. 304), 200 é o tamanho mínimo para um estudo de mercado. Como incentivo aos respondentes que completaram a pesquisa, foram sorteados dois cupons no valor de $R \$ 100,00$ cada para fins de motivação do engajamento dos respondentes na pesquisa.

A partir dos 220 questionários válidos realizou-se análise descritiva e inferencial dos dados coletados. Foram realizados Testes de Independência do Qui-quadrado por meio do software SPSS ${ }^{\circledR}$, com a finalidade de verificar se o perfil do respondente interfere na escolha de medicamento.

\section{APRESENTAÇÃO E DISCUSSÃO DOS RESULTADOS}

A amostra coletada é predominantemente composta por pessoas do sexo feminino (71,4\%), com idade entre 18 e 30 anos, compreendendo 57,7\% do total de entrevistados. A maior parte declarou possuir curso superior completo ou incompleto, representando $53,6 \%$ e $38,6 \%$, respectivamente. Ainda, $57,8 \%$ dos respondentes declararam possuir uma renda familiar entre três e dez salários mínimos por mês.

De acordo com $72,7 \%$ dos respondentes, a compra de medicamentos é preferencialmente realizada perto da residência. Em relação ao tipo de farmácia para realização de compras de medicamentos, $94,1 \%$ dos respondentes declararam preferir farmácias de grandes redes, como Droga Raia, Drogasil, Drogaria São Paulo, Onofre e Pague Menos, em detrimento das farmácias de bairro. Quanto às motivações que levam a preferência por determinado tipo de farmácia pelos respondentes, 38,2\% afirmaram que o principal motivo para escolha é a localização, seguido pelo preço e pela conveniência, com $34,6 \%$ e $16,4 \%$, respectivamente.

Quando questionados sobre o tipo de medicamento escolhido no momento da compra, 62,3\% afirmaram ter preferência por medicamentos genéricos ou similares, 29,1\% por medicamentos de referência (de marca) e 8,6\% declararam ser indiferentes ao tipo de medicamento. Em relação ao conhecimento sobre a diferença entre medicamentos genéricos, similares e de referência, $76,4 \%$ dos respondentes declararam saber a diferença entre os tipos, contra 23,6\% que afirmaram não saber a diferença, sendo que pelo Teste de Independência do Qui-quadrado foi possível verificar que os mais jovens (até 30 anos) tendem a ter uma maior falta de conhecimento sobre a diferença entre os medicamentos genéricos, similares e de referência. Foi possível observar também, pelo mesmo teste, que os respondentes com 
maior poder aquisitivo (acima de 10 salários mínimos) tendem a comprar medicamentos de referência, enquanto os respondentes com menor renda (entre 3 e $5 \mathrm{SM}$ ) tendem a comprar medicamentos genéricos.

O Teste de Independência do Qui-quadrado foi utilizado para atender a um dos objetivos secundários do estudo, que propõe analisar se o perfil socioeconômico do entrevistado interfere na escolha do medicamento. Cabe ressaltar aqui, que o Teste de Independência do Qui-quadrado, aplicado a variáveis nominais, permite inferir os resultados da amostra probabilística para a população, a partir de duas hipóteses concorrentes:

$$
\left\{\begin{array}{l}
H_{0} \text { : as variáveis são independentes } \\
H_{1} \text { : existe relação de dependência entre as variáveis }
\end{array}\right.
$$

Como a amostra deste estudo é não-probabilística, não é possível o uso do teste para inferir sobre a população a partir da amostra. Apesar disso, o teste foi conduzido para buscar evidências de dependência significativa, entre as variáveis de perfil do respondente e as variáveis pesquisadas sobre o seu comportamento no momento da compra de medicamentos genéricos, similares e referência.

Conforme descrito no gráfico 1, considerando os medicamentos genéricos e similares, as principais motivações que levam a preferência por estes tipos, por parte dos respondentes, são: preço, qualidade e confiança, com $84,7 \%, 6,6 \%$ e 5,8\%, respectivamente, e para medicamentos de referência são: qualidade, confiança e recomendação médica, com 54,7\%, $26,6 \%$ e $9,4 \%$, respectivamente.

Gráfico 1: motivações que levam a preferência por determinado tipo de medicamento pelos respondentes genérico ou similar e de referência

\begin{tabular}{|c|c|c|c|c|}
\hline \multirow{2}{*}{$\begin{array}{r}\text { Preço. } \\
\text { Qualidade. }\end{array}$} & $\square 84,7 \%$ & Qualidade. & \multicolumn{2}{|c|}{$54,7 \%$} \\
\hline & $6,6 \%$ & Confiança. & $26,6 \%$ & \\
\hline Confiança. & $5,8 \%$ & Recomendação médica. & $\square 9,4 \%$ & \\
\hline $\begin{array}{l}\text { Recomendação do balconista da } \\
\text { farmácia. }\end{array}$ & $1,5 \%$ & Marca. & $4,7 \%$ & \\
\hline Recomendação médica. & $1,5 \%$ & Recomendação do balconista.. & $3,1 \%$ & \\
\hline Marca. & $0,0 \%$ & Preço. & $1,6 \%$ & \\
\hline Fidelidade. & $\mathbf{0 , 0 \%}$ & Fidelidade. & $\mathbf{0 , 0 \%}$ & \\
\hline Outro(s) motivo(s). & $\mathbf{0 , 0 \%}$ & Outro(s) motivo(s). & $\mathbf{0 , 0 \%}$ & \\
\hline$\square$ Genéric & ou similar. & $\square$ Referê & ncia (de marca) & \\
\hline
\end{tabular}

Fonte: Elaboração própria

Quanto à preferência pelos medicamentos do tipo genérico e similar, os resultados apresentados indicam que preço e qualidade são os principais atributos influenciadores do processo de decisão de compra dos respondentes. Já para os medicamentos de referência, a classe médica, por meio da prescrição, estimula o uso de medicamentos originadores em detrimento dos genéricos e similares, estando atrelado a isso, a percepção de maior qualidade percebida e também maior confiança pelos medicamentos de referência. Esses resultados são semelhantes aos achados de outros estudos que analisaram a decisão de compra desses tipos de medicamentos (FERNANDES, COUTINHO, VALLE , 2011; SANYAL, DATTA, 2011; DUNNE, S, DUNNE, C., 2015; GUTTIER et al., 2016; SANYAL, DATTA, BANERJEE, 2017; FERREIRA et al. (2017). 
Com base no Teste de Independência do Qui-quadrado, foi possível verificar que os respondentes mais velhos (acima de 30 anos) e com maior renda (acima de 10 salários mínimos) tendem a escolher o medicamento de referência baseado na fidelidade ao produto, enquanto que os respondentes mais jovens (até 30 anos) e com menor renda (até 5 salários mínimos) tendem a escolher o medicamento genérico ou similar baseado no preço.

O Shopper Marketing tem por objetivo compreender o comportamento dos clientes, sejam estes os consumidores ou não e as experiências as quais são submetidos no ponto de venda, de forma a analisar os fatores de influência do processo de decisão de compra dos consumidores (CONSOLI; GUISSONI; D`ANDREA, 2011). Logo, entender o comportamento do shopper se faz necessário para identificar quais são os atributos envolvidos no processo de decisão de compra, considerando especificamente o ambiente do ponto de venda (PORTAL ESPM, 2014).

Desta forma, considerando-se o comportamento do shopper, a preferência para compra por um dos tipos de medicamentos - genérico, similar ou de referência, considerando que o respondente estaria realizando a compra para outra pessoa, 59,8\% afirmaram ter preferência por medicamentos genéricos ou similares, 30,8\% por medicamentos de referência e 9,4\% declararam ser indiferentes ao tipo de medicamento. Os resultados observados foram semelhantes aos observados para o cenário dos respondentes como consumidores, cabendo ressaltar aqui que foi possível verificar por meio do Teste de Independência do Qui-quadrado que esses consumidores que fazem compras para outras pessoas tendem a ter idade superior a 30 anos, enquanto que os consumidores mais jovens (até 30 anos) tendem a comprar medicamento para consumo próprio.

Como pode ser visto no gráfico 2, na compra de medicamentos genéricos e similares, as principais motivações que levam a preferência por estes tipos de medicamento por parte dos respondentes quando realizam a compra para outra pessoa (shopper) são: preço, qualidade e confiança, com $74,3 \%, 12,9 \%$ e $8,6 \%$, respectivamente e para medicamentos de referência (de marca), qualidade, confiança e recomendação médica, com 50\%, 33,3\% e 8,3\%, respectivamente. Foi possível observar que os resultados foram semelhantes aos obtidos para o cenário dos respondentes como consumidores.

Gráfico 2: motivações que levam a preferência por determinado tipo de medicamento pelos respondentes (shopper) quando realizam a compra para outra pessoa - genérico ou similar e referência (de marca).

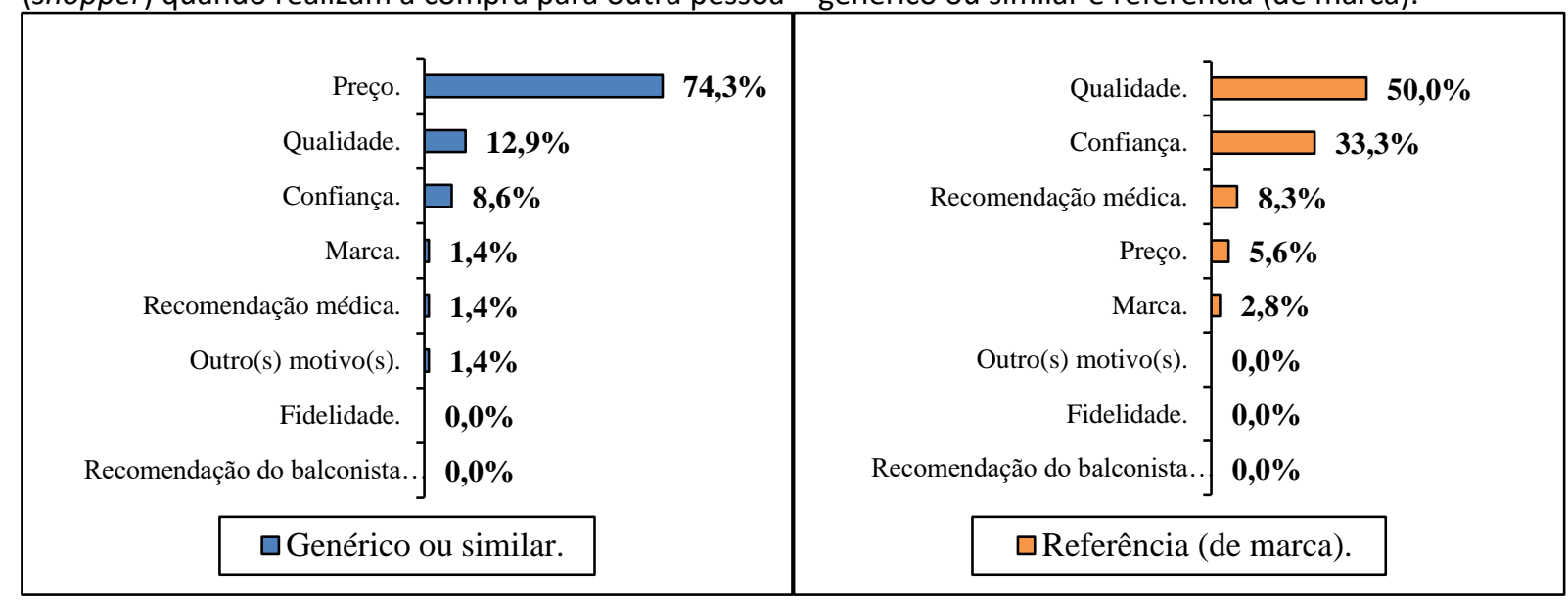

Fonte: Elaboração própria

Analogamente, quanto à preferência pelos medicamentos do tipo genérico e similar, nota-se que preço e qualidade permanecem como os principais atributos que influenciam no processo de decisão de compra dos shoppers. Já para os medicamentos de referência, a classe 
médica, por meio da prescrição, estimula e o uso de medicamentos originadores em detrimento dos genéricos e similares, estando atrelado a isso, a percepção de maior qualidade percebida e maior confiança pelos medicamentos de referência. Tal como dito anteriormente, os resultados são coincidentes aos observados nos estudos realizados por Fernandes, Coutinho e Valle (2011), Sanyal e Datta (2011), Dunne, S e Dunne, C (2015), Ferreira et al. (2017) e Sanyal, Datta e Banerjee (2017).

O gráfico 3 apresenta o nível de influência de profissionais da saúde. Em relação ao nível de influência da recomendação médica e também da recomendação do balconista/atendente da farmácia para escolha do tipo de medicamento, genérico, similar ou de referência, 55,9\% dos respondentes declararam ser alta a influência dos médicos, enquanto $68,2 \%$, afirmou ser de média a alta o nível de influência dos balconistas/atendentes de farmácia para realização desta escolha. Foi possível observar pelo Teste de Independência do Qui-quadrado que os consumidores mais jovens (até 30 anos) e com menos instrução (até superior incompleto) são os que sofrem maior influência dos balconistas/atendentes.

Gráfico 3: nível de influência de profissionais da saúde para escolha do tipo de medicamento - recomendação médica e dos balconistas/atendentes de farmácia.

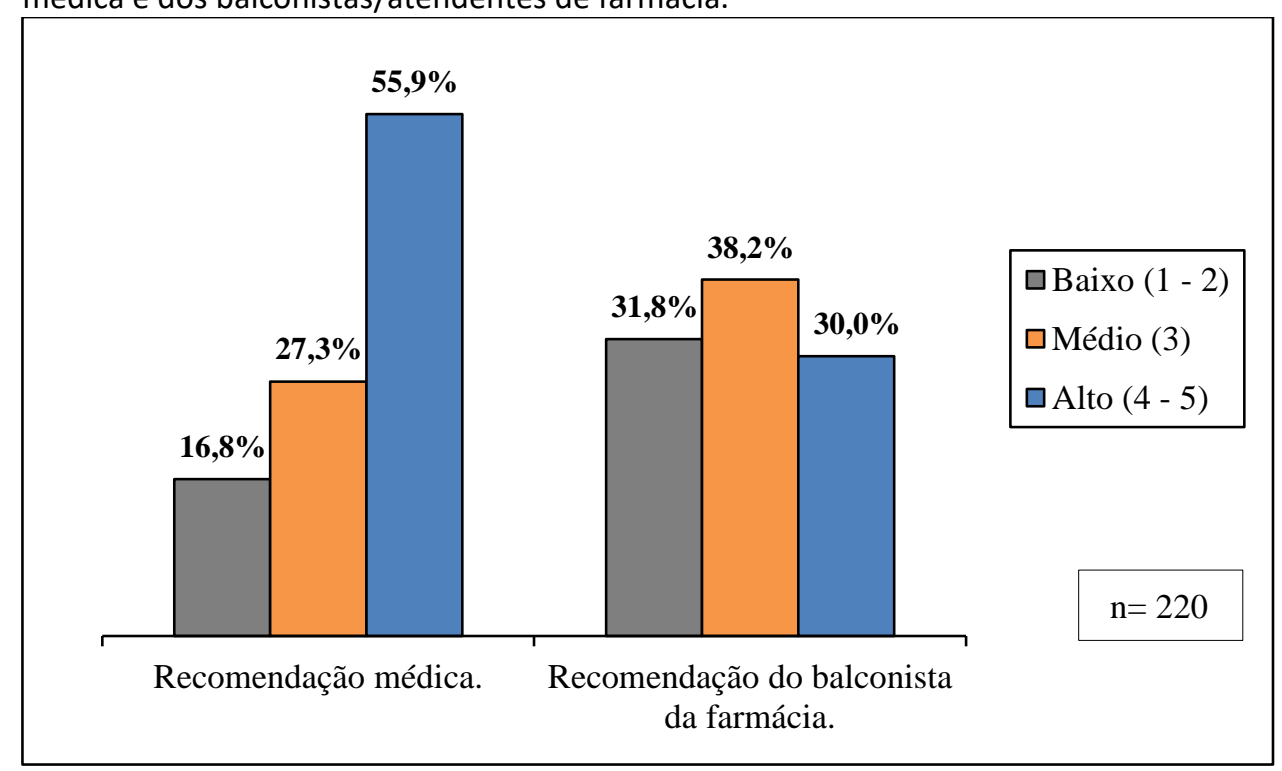

Fonte: Elaboração própria

Deste modo, os profissionais da saúde, sejam estes da classe médica ou funcionários que trabalham como balconistas em farmácias, possuem um grande potencial de influência na decisão dos consumidores pelo tipo de medicamento a ser adquirido, sendo esta influência direcionada aos medicamentos de referência, sendo esses resultados condizentes com os achados de outros trabalhos que também avaliaram essas questões (SANYAL, DATTA, 2011; DUNNE, S., DUNNE, C, 2015; GUTTIER et al., 2016; SANYAL, DATTA, BANERJEE, 2017; FERREIRA et al., 2017).

Por fim, podemos destacar ainda que pelo Teste de Independência do Qui-quadrado foi possível observar que os respondentes, sejam homens ou mulheres, apresentam comportamentos semelhantes no momento da compra de medicamentos genéricos, similares ou de referência, isto é, o comportamento de compra dos respondentes mostrou-se independente do sexo dos mesmos. 


\section{CONSIDERAÇÕES FINAIS}

Como apresentado, diante do cenário de crescimento e das perspectivas para o mercado farmacêutico, com destaque para o desempenho dos medicamentos genéricos e similares, definiu-se como objetivo para este estudo, a análise da relevância dos atributos marca, qualidade e preço no processo de decisão de compra dos consumidores de medicamentos genéricos, similares e de referência na cidade de São Paulo e nos municípios da região metropolitana.

Os resultados indicam que a maioria dos respondentes da pesquisa têm conhecimento da diferença entre os medicamentos genéricos, similares e de referência. Quanto as motivações que levam a preferência por esses tipos de medicamentos, preço, qualidade e confiança são os principais motivadores para a compra de medicamentos genéricos e similares, e qualidade, confiança e recomendação médica para os medicamentos de referência. Analisadas as motivações quando os respondentes realizam a compra para outra pessoa (shopper), padrão similar ao citado anteriormente também foi observado.

Podemos destacar que o preço no caso dos medicamentos genéricos e qualidade no caso dos medicamentos de referência são os atributos que tiveram maior influência na decisão de compra desses produtos. No caso dos medicamentos de referência, a confiança também foi considerada um atributo bastante relevante. Em contrapartida, a marca teve um impacto muito pequeno na decisão de compra desses medicamentos por parte dos consumidores e/ou shoppers.

Em paralelo, a influência de profissionais da saúde na intenção de compra dos consumidores, quanto à recomendação médica ou à recomendação do balconista da farmácia, também se mostrou expressiva, conforme indicado pelos dados do estudo, sendo estes responsáveis pela melhor disseminação das informações e incentivo ao uso de medicamentos genéricos e similares, visando assim à ampliação do acesso de medicamentos a maior parte da população.

É importante ser enfatizado que esses achados são condizentes com os resultados de diferentes estudos realizados anteriormente por Fernandes, Coutinho e Valle (2011), Sanyal e Datta (2011), Guttier et al. (2016), Ferreira et al. (2017) e Sanyal, Datta e Banerjee (2017) que avaliam variáveis similares as utilizadas neste trabalho, mas realizadas com outros perfis de consumidores e contextos de mercado, o que reforça o impacto das mesmas no processo de decisão de compra de medicamentos genéricos, similares e de referência.

Quanto a análise da influência do perfil socioeconômico no processo de decisão de compra, podemos observar que, para a amostra pesquisada, idade, renda e grau de instrução do consumidor interferem na escolha do medicamento, enquanto o sexo do respondente parece não interferir.

Os resultados deste estudo merecem ser analisados pelas empresas envolvidas no mercado brasileiro de medicamentos. As fabricantes de medicamentos devem avaliar suas estratégias comerciais considerando os principais fatores que impactam a decisão de compra dos consumidores, sendo preço para os medicamentos genéricos e similares, e qualidade e confiança para os medicamentos de referência. Além disso, essas empresas também devem avaliar quais serão as ações na divulgação dos medicamentos junto a classe médica, assim como nos profissionais das farmácias.

Com relação às limitações deste estudo, temos a amostragem não probabilística por conveniência e a restrição do público-alvo estudado ter sido somente os consumidores de medicamentos na grande São Paulo. Tais limitações impossibilitam a generalização dos 
resultados obtidos para a população em geral, porém não invalidam as análises realizadas e os achados para a amostra coletada. São sugestões para minimização destas limitações para próximos estudos a realização de uma amostragem probabilística por cotas e a expansão do público-alvo do estudo a nível estadual ou nacional.

\section{REFERÊNCIAS}

ALMEIDA, A. R. D.; BOTELHO, D. Confiança, qualidade ou valor percebido: o que influencia a satisfação na compra de um imóvel? In: Encontro da Associação Nacional dos Programas de Pós-Graduação em Administração - EnANPAD, 29., Brasília/DF. Anais [...]. Brasília/DF: ENANPAD, 2005.Disponível em:

http://www.anpad.org.br/diversos/down_zips/9/enanpad2005-mktb-2262.pdf. Acesso em: 30 out. 2017.

ALISKI, A. Anvisa determina suspensão de lote do medicamento Omeprazol. Revista Exame 2014. Disponível em: <http://exame.abril.com.br/brasil/noticias/anvisadeterminasuspensaode-lote-do-medicamento-omeprazol>. Acesso em: 31 ago. 2017.

ANVISA. Legislação: Leis. Lei no 9.787, de 10 de fevereiro de 1999. Disponível em: < http://www.anvisa.gov.br/hotsite/genericos/legis/leis/9787.htm>. Acesso em: 31 ago. 2017.

ANVISA. Legislação. Resoluções. Resolução - RDC no 135, de 29 de maio de 2003. Disponível em: <http://www.anvisa.gov.br/hotsite/genericos/legis/resolucoes/2003/135_03rdc.htm>. Acesso em: 31 ago. 2017.

BARATA-SILVA, C.; HAUSER-DAVIS, R. A.; SILVA, A. L. O.; MOREIRA, J. C. Desafios ao controle da qualidade de medicamentos no Brasil. Caderno de Saúde Coletiva, v. 25, n. 3, p. 362-370, 2017. DOI: http://dx.doi.org/10.1590/1414-462x201700030075.

BLACKWELL, R. D.; MINIARD, P. W.; ENGEL, J. F. Comportamento do consumidor. São Paulo: Thomson Learning, 2005.

BLATT, C. R.; TRAUTHMAN, S. C.; SCHMIDT, E. H.; MARCHESAN, S.; SILVA, L. M.; MARTINS, J. L. Conhecimento popular e utilização dos medicamentos genéricos na população do município de Tubarão, SC. Ciência e Saúde Coletiva Online, v. 17, n. 1, p. 79-87, 2012.DOI: http://dx.doi.org/10.1590/S1413-81232012000100011.

COBRA, M; BREZZO, R. O novo marketing. Rio de Janeiro: Elsevier, 2010.

CONSOLI, M.A.; GUISSONI, L.A.; D`ANDREA, R. Shopper Marketing: a nova estratégia integrada de marketing para a conquista do cliente no ponto de venda. Editora Atlas, São Paulo, SP, Brasil, 2011.

CUNHA, A. L. $O$ valor da marca para o consumidor final a partir do conceito de brand equity no mercado de iogurtes. Dissertação (Mestrado) - Escola de Administração, Universidade Federal do Rio Grande do Sul, Porto Alegre, 1997. 
DUNNE, S. S.; DUNNE, C. P. What do people really think of generic medicines? A systematic review and critical appraisal of literature on stakeholder perceptions of generic drugs. BioMed Central Medicine, v. 13, n. 173, p. 1-27, 2015.DOI: https://doi.org/10.1186/s12916015-0415-3

ERDEM. T.; SWAIT, J. Brand equity as a signaling phenomenon. Journal of consumer Psychology, v. 7, n. 2, p. 131-157, 1998.

FERNANDES, J. A.; COUTINHO, J. V.; VALLE, M. G. Aceitação do medicamento genérico em diferentes níveis de escolaridade e renda familiar do Distrito Federal. Cenarium Pharmacêutico, v. 4, n. 4, 2011.

FERREIRA, V. L.; VEIGA, C. R. P.; KUDLAWICZ-FRANCO, C.; SCALERCIOA, P.; RAMIRESA, Y.; PONTAROLOA, R.; CARVLHO, D. M.W. VEIGA, C. P. Generic drugs in times of economic crisis: Are there changes in consumer purchase intention? Journal of Retailing and Consumer Services, v. 37, p. 1-7, 2017. DOI: https://doi.org/10.1016/j.jretconser.2017.02.008

FITZGERALD, C. L.; JACOBSON, M. P. Generic substitution of levetiracetam resulting in increased incidence of breakthrough seizures. Annals of Pharmacother, v. 45, n. 5, 2011.DOI: https://doi.org/10.1345/aph.1P765

FONSECA, E. M. Reforming pharmaceutical regulation: a case study of generic drugs in Brazil. Policy Soc, n. 33, p. 65-76, 2014.DOI: https://doi.org/10.1016/j.polsoc.2014.03.004

GAITHER, C. A.; KIRKING, D. M.; ASCIONE, F. J.; WELAGE, L. S. Consumers' views on generic medications. Journal of the American Pharmaceutical Association, v. 41, n. 5, p. 729-736, 2001. DOI: https://doi.org/10.1016/S0003-0465(15)33129-3

GASPAR, J. F. T. Comportamento do consumidor na compra e utilização do medicamento genérico. Dissertação de Mestrado. Portugal-Universidade de Coimbra, 2016.

GILL, L.; HELKKULA, A.; COBELLI, N.; WHITE, L. How do customers and pharmacists experience generic substitution? International Journal of Pharmaceutical and Healthcare Marketing, v. 4, n. 4, p. 375-395, 2010. DOI: https://doi.org/10.1108/17506121011095218

GUTTIER, M. C.; SILVEIRA, M. P. T.; LUIZA, V. L.; BERTOLDI, A. D. Percepção, conhecimento e uso de medicamentos genéricos no Sul do Brasil: o que mudou entre 2002 e 2012? Caderno de Saúde Pública, v. 32, n. 7, 2016. https://doi.org/10.1590/0102-311X00070215

HAIR JR, J.; BABIN, B.; MONEY, A. H.; SAMOUEL, P. Fundamentos de métodos de pesquisa em administração. 6. ed. Porto Alegre: Bookman, 2009.

HAN, S.; GUPTA, S.; LEHMANN, D. Consumer price sensitivity and price thresholds. Journal of Retailing, v. 77, p. 435-456, 2011.DOI: https://doi.org/10.1016/S0022-4359(01)00057-4

HOEFFLER, S.; KELLER K. The Marketing Advantages of Strong Brands. Brand Management, v. 10, n. 6, p. 421-445, 2003. DOI: https://doi.org/10.1057/palgrave.bm.2540139 
INTERFARMA - ASSOCIAÇÃO DA INDÚSTRIA FARMACÊUTICA DE PESQUISA. Guia Interfarma 2016. Disponível em:< http://www.interfarma.org.br/guia/guia_2016/ apresentacao>. Acesso em: 30 jun. 2017.

KAYTAZ, M., GUL, M. C. Consumer response to economic crisis and lessons for marketers: the Turkish experience. Journal of Business Research, v. 67, n. 1, p. 2701-2706, 2014.DOI: https://doi.org/10.1016/j.jbusres.2013.03.019

KASWENGI, J.; DIALLO, M. F. Consumer choice of store brands across store formats: a panel data analysis under crisis periods. Journal of Retailing and Consumer Services, v. 23, p. 7076, 2015. DOI: https://doi.org/10.1016/j.jretconser.2014.12.003

KELLER, K. L.; MACHADO, M. Gestão estratégica de marcas. São Paulo: Pearson PrenticeHall, 2006.

KINNEAR, T. C.; TAYLOR, J. R. Marketing Research. New York: McGraw-Hill, 1999.

KOBAYASHI, E.; KARIGOME, H.; SAKURADA, T.; SATOH, N.; UEDA, S. Patients' attitudes towards generic drug substitution in Japan. Health Policy, v. 99, p. 60-65, 2011. DOI: https://doi.org/10.1016/j.healthpol.2010.07.006

KOTLER, P.; KELLER, K. L. Administração de marketing. 14. ed. São Paulo: Pearson Education do Brasil, 2012.

LIMA, M. V. V.; STEINER NETO, P. J. A influência da qualidade percebida e das emoções como antecedentes da satisfação: um estudo com consumidores de serviços hoteleiros. Revista FSA, v. 10. n. 4, p. 1-19, 2013.

MALHOTRA, N. K. Pesquisa de marketing: uma orientação aplicada. 6. ed. Porto Alegre: Bookman, 2012.

MINTZES. B.; BARER, M. L.; KRAVITZ, R. L.; BASSETT, K.; LEXCHIN, J.; KAZANJIAN, A.; EVANS, R. G.; PAN, R.; MARION, S. A. How does direct-to-consumer advertising (DTCA) affect prescribing? A survey in primary care environments with and without legal DTCA. CMAJ, $n$. 169, n. 5, p. 405-412, 2003.

MOSS, G. Pharmaceuticals - where's the Brand Logic? Branding Lessons and Strategy, Pharmaceutical Products Press. The Haworth Press Inc, New York, 2007.

MURRAY, E.; LO, B.; POLLACK, L.; DONELAN, K.; CATANIA, J.; WHITE, M.; ZAPERT, K.; TURNER, $R$. The impact of health information on the internet on the physician-patient relationship: patient perceptions. Arch Intern Med, v. 163, n. 14, p. 1727-34, 2003.

DOI:10.1001/archinte.163.14.1727

PRICEWATERHOUSECOOPERS (PWC). O Mercado de Serviços de Saúde no Brasil, 2014. Disponível em: <https://www.pwc.com.br/pt/publicacoes/setoresatividade/assets/saude/healthcare-port.pdf >. Acesso em: 15 maio 2017 
PORTAL ESPM. Shopper Marketing: A Influência no Momento da Compra. ESPM. Núcleo de Varejo. Retail. Lab. 2014. Disponível em: <http://varejo.espm.br/shopper-marketing-ainfluencia-no-momento-da-compra>. Acesso em: 29 dez. 2017.

NAGLE, T. T; HOGAN, J. E. Estratégia e táticas de preço: um guia para crescer a lucratividade. 4. ed. São Paulo: Pearson, 2007.

SAMAOUI, F.; KILANI, F. A.; TOUZANI, M. Country-of-origin versus brand: consumers' dilemma when choosing between generic and branded drugs in emerging countries. Journal of Product \& Brand Management, v. 25, n. 2, p. 148-159, 2016. DOI 10.1108/JPBM04-2014-0553

SANSGIRY, S. S.; BHOSLE, M; POPE, N. Consumer Perceptions Regarding Generic Drug Substitution: An Exploratory Study. Journal of Pharmaceutical Marketing \& Management, v. 17, n. 1, p. 77-91, 2005. DOI: https://doi.org/10.3109/J058v17n01_06

SANTOS, C. P.; ROSSI, C. A. V. Os Antecedentes da Confiança do Consumidor em Episódios envolvendo Reclamações sobre Serviços. In: 26ㅇ Encontro da Associação Nacional dos Programas de Pós-Graduação em Administração - EnANPAD, 2002, Salvador/BA. Anais... Salvador/BA: Associação Nacional dos Programas de Pós-Graduação em Administração. 2002.

SANYAL, S. N.; DATTA, S. K. The effect of perceived quality on brand equity: an empirical study on generic drugs. Asia Pacific Journal of Marketing and Logistics, v. 23, n. 5, p. 604625, 2011. DOI: https://10.1108/13555851111183057

SANYAL, S. N.; DATTA, S. K.; BANERJEE, A. K. Factors influencing prescribing decisions among physicians: an empirical study on generic drugs. International Journal of Pharmaceutical and Healthcare Marketing, v. 11, n. 4, p. 330-360, 2017. DOI: https://10.1108/IJPHM-06-2016-0031

SERRALVO, F. A. Gestão de marcas no contexto brasileiro. São Paulo: Saraiva, 2008.

SINDICATO DA INDÚSTRIA DE PRODUTOS FARMACÊUTICOS NO ESTADO DE SÃO PAULO (SINDUSFARMA). Indicadores setoriais da indústria farmacêutica. Disponível em:< http://sindusfarma.org.br/cadastro/index.php/site/app_indicadores $>$. Acesso em: 20 jun. 2017.

SKIPPER, N.; VEJLIN, R. Determinants of generic vs. brand drug choice: Evidence from population-wide Danish data. Social Science \& Medicine, v. 130, p. 204-215, 2015.DOI: https://doi.org/10.1016/j.socscimed.2015.01.013

SOLOMON, M. R. O comportamento do consumidor: comprando, possuindo e sendo. 11. ed. Porto Alegre: Editora A, 2016. 
SPITERI, J. M.; DION, P. A. Customer value, overall satisfaction, end-user loyalty, and market performance in detail intensive industries. Industrial Marketing Management v. 33, p. 675687, 2004. DOI: https://doi.org/10.1016/j.indmarman.2004.03.005

SOUSA, C. V.; MESQUITA, J. M. C.; LARA, J. E. Análise da decisão de compra de medicamentos frente à existência de produtos substitutos: um estudo no município de Belo Horizonte. Ciência \& Saúde Coletiva, v. 11, p. 3311-3320, 2013.

URBANY, J. E.; BEARDEN, W. O.; KAICKER, A.; DE BORRENO, M. S. Transaction utility effects when quality is uncertain. Academy of Marketing Science Journal, v.25, n.1, p.45-55, 1997.DOI: https://doi.org/10.1007/BF02894508

YANAZE, M. H. Gestão de Marketing e Comunicação: avanços e aplicações. São Paulo: Saraiva, 2011.

ZEITHAML, V. A. Consumer Perceptions of Price, Quality, and Value: A Means-End Model and Synthesis of Evidence. Journal of Marketing, v. 52, n. 3, p. 2-21, 1988.DOI: https://doi.org/10.1177/002224298805200302

ZERBINI, C.; VERGURA, D. T; LUCERI, B. Consumers' intention to buy generic drugs: Evidences from the Italian setting. Mercati \& Competitività, v. 1, p. 159-176, 2017.

DOI: https://doi.org/10.3280/MC2017-001009

ZIKMUND, W. G.; BABIN, B. J. Princípios da pesquisa de marketing. 2. ed. São Paulo:

Cengage Learning, 2012. 